liver was somewhat congested. The spleen was soft and congested. The kidneys were very much congested. The uterus, ovaries, and Fallopian tubes, were much congested. (She was menstruating at the time of her death.) The superficial vein of the brain, as those of the medulla oblongata, were turgid with dark blood. There was a slight amount of serum beneath the arachnoid (which was somewhat opaque here and there), especially in the basilar fossa. The brain-substance itself was perfectly normal. The larynx, trachea, and œosophagus were healthy, and contained no traces of any food in them.

REMARKS. This case is interesting in medical jurisprudence, in two points of view : first, for the unusual precision with which the entire suddenness of the death could be fixed; second, for the total absence of any post mortem appearances usually found in cases of sudden death (such as heart-disease, bursting of aneurisms, etc.). In the Annales d'Hygiène Publique for 1838 will be found a paper by Devergie entitled "De la Mort Subite", in which, with other causes of sudden death, he signalises that by syncope, which he had found to occur three times in forty cases. Dr. Tourdes reported to the scientitic congress meeting held at Strasbourg in 1842, that he had met with this cause of sudden death once in twenty-six cases. It appears to be identical with the "idiopathic asphyxia" of Chevalier. Devergie, in the paper above referred to, gives the following as the post mortem appearances he found in his three cases of death from syncope. " 1 . The absence of all congestion of the organs. 2. The normal state of all the organs. 3. The existence of about an equal quantity of blood in the right and left cavities of the heart, regard being had to their respective sizes. 4. Perhaps the coagulation of the blood in the fibrinous state." As another cause of sudden death Devergie further indicates that arising from cerebral conges tion, which may, he says, be limited to the meninges. In illustration of this, he relates the case of a man aged 63 , who, having only complained of a slight headache in the morning, took a good breakfast, after which he slept for two bours, and, on awaking, suddenly died. No congestion of the brainsubstance was found; there was an old apoplectic clot in the left corpus striatum; there was great congestion of the meninges of the brain and spinal cord.

Directing attention now to the condition of the stomach in our case, we may naturally ask ourselves whether the undigested meal, or the patch of injection on its coats, may have had anything to do with the cause of death? As regards the latter, no one would, I think, after the researches of Dr. Yellowly and others, attach any great independent value to mere vascularity of the gastric mucous membrane. The undigested meal may perhaps possess rather more significance. The deceased seems to have been in the habit of "bolting" her meals very rapidly. The stewed steak found in her stomach, I am informed, she partook of stealthily - and therefore probably hastily-before her proper dinner, shortly before death.

Dr. Taylor, at p. 152 of the second edition of his work on Poisons, has cited several instances of persons dying quite suddenly, with nothing to account for death but a distension of the stomach ; but I noticed no such distension in the present case. We may not unreasonably connect these conditions of the stomach with the syncopal affection. Professor Weber has shown that irritation of the pneumogastrics will stop the heart's action; and my distinguished friend and colleague, Dr. Brown-Séquard, has further shown that crushing the semilunar ganglia has a similar effect on the heart, provided the great splanchnic and pneumogastric nerves remain intact.

I should feel disposed to regard the present case as one of a mixed nature, presenting some of the signs of syncopal asphyxia, others of apoplexy. Explain it, however, as we may, it still remains one of considerable interest, proving as it does that, under certain (as yet very imperfectly understood) circumstances, death may occur suddenly, without leaving any traces at all adequate (in our present state of knowledge) to account for the fatal result.

\section{CONNECTION BETWEEN CANCER AND FRAGILITAS OSSIUM.}

By W. McCheane, Esq., Liverpool.

THE following case may perhaps be worth recording, as illustrative of the connection between cancer and fragilitas ossium.

R. W., a female, aged 68 , had been under my care for some time for a scirrhous tumour in the right breast, from which situation a similar tumour was formerly removed. Of late, she had been altogether confined to bed with guawing pains in the limbs, but more especially in the lower extremities, and in a state of general cachexia.

On the 1st instant, during a fit of coughing, she felt a something give way in the region of the right hip, which sensation was immediately followed by severe pain in the part and down the whole limb. On examination, I found a fracture of the neck of the thigh-bone, which injury she survived only a week.

Some months ago she fractured two ribs with lifting a pan off the fire; the bones, however, readily united.

\section{Cramsactions of 通rand hes.}

\section{BIRMINGHAM AND MIDLAND COUNTIES B RANCH.}

ON THE PATHOLOGY OF LEAD-COLIC.

By Willoughby F. WADE, M.B., Physician to the Queen's Hosnital and to the General Dispensary; Professor of the Practice of Physic in the Queen's College, Birmingham.

[Read April 19th, $1 \$ 60$. .]

THE received opinion that this painful disorder depends upon some perverted action of the colon, as its name implies, has already had its antagonists. When we come to inquire a little more closely what this perverted action is, we find that no satisfactory answer can be given. Some contend for an empty and contracted condition of the gut, others for a distension by gas or fæces.

Dr. Copland says that in his cases distension was as frequent as retraction, owing evidently to inflation and fæcal engorgement of the colon, the cour'se of which could be distinctly traced under the abdominal parietes. De Haen and Merat found contraction of the colon and cæcum in all the cases they examined. But, as Dr. Watson judiciously remarks, "with regard to the contraction of the large intestine in these cases, we must not be too ready to attribute it to spasm, for the bowel, when empty, is apt to be contracted." Andral details six cases in which no such contractions were found. Indeed Andral, Louis, and Sir George Baker, concur in describing the intestines as being normal throughout their whole extent. I doubt very much whether an unopposed contraction of a hollow muscular canal can be attended with pain. It is the vain endeavour to shorten the muscular tissue, and the resistance offered by an incompressible material, that causes the pain in biliary calculus and ordinary crapulous or flatulent colic. An empty intestine might, I think, go on contracting till its calibre was obliterated before it produced pain. 'The after-pains of labour do not offer any necessary objection to this view; for the contraction of one layer of fibres can be well resisted by the large mass of inactive ones. Besides, they often depend on the presence of clots. On the other hand, did the pain of lead-colic depend upon the presence of flatus, I cannot conceive how it is that this should not, in such cases, be readily removed, for a time at least, by opiates and carminatives, as happens in ordinary flatulence. If, again, it depended upon retained fæces, the removal of them should remove the pain. But the operation of the bowels is by no means necessarily followed by this relief. It is, indeed, true that the two often coincide, but this is quite as easily explicable in another way, as we shall see directly. The retraction of the abdominal parietes, so constantly noticed in this form of the complaint, is by no means so constantly observed in other varieties of colic.

The pathology of lead-colic is then, I submit, unsatisfactory and vague as at present taught.

Various pathologists of distinction have been disposed to refer the symptoms to cramp of the external abdominal muscles, instead of to the intestines at all. Giacomini first broached this notion, and M. Briquet of La Charite has more lately revived this view, which he supports with skill and vigour. The existence of cramp in these muscles has been recognised by those who are entirely committed to the generally accepted pathology. Thus Dr. Copland says, " the voluntary muscles often become so sore that they cannot bear the slightest pressure; and the pain frequently alternates between the stomach and bowels and the external muscles." Besides 